Caglioti, M. T. \& Sermonti, G. (1956). J. gen. Microbiol. 14, 38-46

\title{
A Study of the Genetics of Penicillin-producing Capacity in Penicillium chrysogenum
}

\author{
BY MARIA T. CAGLIOTI AND G. SERMONTI \\ International Research Centre for Chemical Microbiology, Istituto \\ Superiore di Sanità, Rome, Italy
}

SUMMARY: Four non-penicillin-producing $(p)$ strains were isolated from the strain 47. 1564 Wis. of Penicillium chrysogenum and used in this work. Seven heterokaryons and seven heterozygous diploids were synthesized between the four $p$ strains and one penicillin-producing $(+)$ strain. All these strains were further marked by nutritional requirements and differences in colour of the conidia. None of the heterokaryons and diploids between non-producing strains produced any appreciable amount of penicillin; all the heterokaryons and diploids between a producing and a non-producing strain did produce penicillin in amounts of the same order as those of the producing strain. The four $p$ mutations behave like alleles: $p$ is recessive to the wild condition. Linkage of the allele $p$ to $y$ and to $c y$ may be deduced from data of the somatic segregation from a heterozygous diploid.

Bonner (1947) was unable to restore penicillin production in non-penicillinproducing mutants by growing numerous pairs of such strains in mixed cultures. Using parasexual recombination in Penicillium chrysogenum (Pontecorvo \& Sermonti, 1953, 1954; Sermonti, 1954a, $b$ ) it is now possible to analyse heterokaryotic, heterozygotic and possibly allele interactions between mutants differing in penicillin production. The present paper reports an investigation to this end.

\section{METHODS}

Unless otherwise stated, the techniques, media, etc., used in the present work are the routine ones for genetics and fermentation researches in Penicillium chrysogenum, as published in detail elsewhere (Sermonti, 1954a, $b$; Camici, Sermonti \& Chain, 1952). Penicillin production on agar was obtained with the basal medium of Jarvis \& Johnson (1950) or the ' $E$ ' medium of Raper, Dorothy \& Coghill (1944). Small plugs of agar were removed close to the colony to be tested and placed on agar inoculated with a test organism (Raper et al. 1944). Only presence or absence of the inhibition halo was taken into account. Penicillin production in submerged culture was tested by a modification of the cylinder-plate method of Schmidt \& Moyer (1944). In both tests the I.C.I. strain of Bacillus subtilis was used.

Mutant production. All the mutants used in the course of the work were derived from strain 47.1564 Wis. of Penicillium chrysogenum, which will be referred to as the wild type. Mutants were isolated after ultraviolet (UV) irradiation of the conidia in a dosage which gave $95 \%$ mortality.

Colour mutants were identified visually among the colonies which grew from the surviving conidia. Nutritional mutants (auxotrophs) were identified 
by the total isolation method (Fries, 1948). Non-penicillin-producing strains were identified by transferring single colonies grown from irradiated conidia to small Petri dishes and then testing the cultures for penicillin production.

Table 1 gives a list of the mutant strains used, with their characteristics.

Table 1. Characteristics and origins of the mutants employed in the course of the research

$\begin{array}{clc}\text { Strain symbol* } & \text { Parent strain } & \text { Mutagenic agent } \\ 97 p_{1} & 47.1564 \text { Wis. } & \text { Ultraviolet } \\ 99 p_{2} & 47.1564 \text { Wis. } & \text { Ultraviolet } \\ 15 y_{1} & 47.1564 \text { Wis. } & \text { Ultraviolet } \\ 20 w & 47.1564 \text { Wis. } & \text { Mustard gas } \dagger \\ 123 y_{2} p_{1} & 97 p_{1} & \text { Ultraviolet } \\ 42 y_{3} p_{2} & 99 p_{2} & \text { Ultraviolet } \\ 92 p_{3} y_{1} & 15 y_{1} & \text { Ultraviolet } \\ 46 \mathrm{me} w & 20 w & \text { Ultraviolet } \\ 124 \mathrm{hy} y_{2} p_{1} & 123 y_{2} p_{1} & \text { Ultraviolet } \\ 122 \mathrm{hy} y_{3} p_{2} & 42 y_{3} p_{2} & \text { Ultraviolet } \\ 65 \mathrm{pr} y_{3} p_{2} & 42 y_{3} p_{2} & \text { Ultraviolet } \\ 63 \mathrm{cy} p_{3} y_{1} & 92 p_{3} y_{1} & \text { Ultraviolet } \\ 121 p_{4} \text { me } w & 46 \mathrm{me} w & \text { Ultraviolet }\end{array}$

* The strain symbols consist of a code number followed by symbols whose interpretation is as follows: $p=$ absence of penicillin production; $y=$ yellow colour of conidia ; $m e=$ methionine requirement; $h y=$ hypoxanthine or adenine requirement; $p r=$ proline requirement; $c y=$ cysteine or methionine requirement. Mimic mutants of similar phenotype representing different mutations are distinguished by a subscript.

$\dagger$ According to the technique of Stahmann \& Stauffer (1947).

Auxotrophic mutants appeared at an average rate of rather less than $1 \%$ of the survivors; mutants not producing penicillin $(p)$ at a rate of about $0.25 \%$.

The four $p$ mutants studied were chosen from a total of ten isolated after testing 3962 colonies which had survived UV irradiation. This kind of mutant was obtained by Bonner (1947) at the rate of $0.2 \%$ after UV irradiation and at the rate of about $0.5 \%$ by Roegner, Stahmann \& Stauffer (1954) by the use of methyl-bis-( $\beta$-chloroethyl)amine.

All the non-penicillin-producing strains used in this work were checked for non-production in submerged culture. Strain $121 p_{4}$ produced about half a penicillin unit/ml.; strains $97 p_{1}$ and $99 p_{2}$ produced smaller quantities which cannot be measured with precision, and strain $92 p_{3} y_{1}$ produced no penicillin at all, compared with $250 \mathrm{u}$./ml. for the wild type control. No appreciably higher penicillin yields were obtained in later tests, even when better yields were obtained from the control.

\section{RESULTS}

\section{Synthesis of balanced heterokaryons}

Nine balanced heterokaryons (Table 2) were obtained with the last six marked strains listed in Table 1 . The combinations were devised to combine the $p$ markers two by two in all possible ways, and two further combinations 
were those of a normal penicillin-producing strain with each of two strains with different $p$ markers.

Table 2. Balanced heterokaryons and the media used to obtain them*

Balanced heterokaryon
$63 c y p_{3} y_{1}+46$ me w
63 cy $p_{3} y_{1}+65$ pr $y_{3} p_{2}$
$63 c y p_{3} y_{1}+122$ hy $y_{3} p_{2}$
$63 c y p_{3} y_{1}+121 p_{4}$ me w
$63 c y p_{3} y_{1}+124$ hy $y_{2} p_{1}$
$124 h y y_{2} p_{1}+65$ pr $y_{3} p_{2}$
124 hy $y_{2} p_{1}+121 p_{4}$ me w
65 pr $y_{3} p_{2}+121 p_{4}$ me w
65 pr $y_{3} p_{2}+46$ me w

Medium $\dagger$
MM + methionine
MM + methionine + proline
A corn steep medium $\ddagger$
MM + methionine
MM + methionine + adenine
MM + adenine + proline
MM + adenine + methionine
MM + proline + methionine
MM + proline + methionine

* Balanced heterokaryons were selected as tufts of better growing mycelium in the zone of contact between two colonies of different types.

$\dagger$ Additions to minimal medium (MM) for growth of mutants were made at the following concentrations (mg./1.): DL-methionine, 10; L-proline, 10 ; adenine, 1.25 .

$\ddagger$ MM supplemented by $1 \%$ (w/v) corn steep liquor.

Heterokaryons were synthesized by the technique developed by Sermonti (1954a) for Penicillium chrysogenum. The nine clones synthesized in this way were shown to be heterokaryons by their ability to give rise to heterozygous diploids. This was further confirmed for some of them by the reappearance of the parent types after plating their conidia on nutritionally complete medium (CM) $(63+121 ; 65+121$ and $46+63)$; by absence of growth from conidia and occurrence of growth from mycelium transfers on minimal medium (MM) $(63+65 ; 63+122 ; 63+121 ; 63+124 ; 65+121$ and $46+65)$; and by recovery of the parent types from conidia of colonies derived after the isolation of single hyphae $(46+63)$.

\section{Selection of heterozygous diploids}

A list of heterozygous diploids is given in Table 3; each diploid was obtained from one of the heterokaryons listed in Table 2, either after plating conidia on MM medium or as sectors from heterokaryon colonies (Roper, 1952). All the heterozygous diploid strains were purified by single conidium isolation with a micromanipulator.

The ability of the strains isolated to give rise to segregants carrying the recessive characters of the parent strains, either combined or in isolation (see Table 7), constitutes a definite demonstration of their being heterozygous diploids.

\section{Characteristics of heterokaryons and of heterozygous diploids}

The general properties of heterokaryons and heterozygous diploids have been the subject of investigations reported in previous papers. Colour of the sporing surface and presence or absence of penicillin production alone will here be described in detail.

Colour. The colour of the sporing colony surface in the case of heterokaryons is intermediate between those of the parent strains (see Table 4). The present 
observations confirm those already reported by Pontecorvo \& Sermonti (1954) and by Sermonti (1954a). The sporing surfaces of heterozygous diploids are coloured various shades of green, i.e. similar to the colour of the wild type: the diploid 63 cy $p_{3} y_{1} / 124 h y y_{2} p_{1}$ is an exception to this rule in having yellow

Table 3. Heterozygous diploids obtained from balanced heterokaryons

(a) Diploids obtained by plating conidia of heterokaryons on minimal medium

\begin{tabular}{|c|c|c|c|c|}
\hline \multirow[b]{2}{*}{ Heterozygous diploids* } & \multicolumn{2}{|c|}{$\begin{array}{l}\text { No. of conidia of the } \\
\text { heterokaryon plated }\end{array}$} & \multirow{2}{*}{$\begin{array}{c}\text { No. of } \\
\text { diploids } \\
\text { obtained }\end{array}$} & \multirow{2}{*}{$\begin{array}{c}\text { Ratio of } \\
\text { conidia plated : } \\
\text { diploids } \\
\text { obtained }\end{array}$} \\
\hline & Total & Per dish & & \\
\hline VI $63 c y p_{3} y_{1} / 65 p r y_{3} p_{2}$ & $18 \cdot 8 \times 10^{6}$ & $13.5 \times 10^{5}$ & 1400 & $13,400: 1$ \\
\hline IX $63 c y p_{3} y_{1} / 122 h y y_{3} p_{2}$ & $9.8 \times 10^{6}$ & $6 \cdot 1 \times 10^{5}$ & 5000 & $1,960: 1$ \\
\hline XIII $63 c y p_{3} y_{1} / 121 p_{4}$ me w & $0.36 \times 10^{6}$ & $0.3 \times 10^{5}$ & 6 & $60,000: 1$ \\
\hline $\mathrm{XV} 63$ cy $p_{3} y_{1} / 124 h y y_{2} p_{1}$ & $1.5 \times 10^{6}$ & $2 \times 10^{5}$ & 622 & $2,410: 1$ \\
\hline
\end{tabular}

(b) Diploids obtained as green sectors from balanced heterokaryons

VI $63 c y p_{3} y_{1} / 65$ pr $y_{3} p_{2}$
VII $63 c y p_{3} y_{1} / 46$ me w
VIII $124 h y y_{2} p_{1} / 65 p r y_{3} p_{2}$
IX $63 c y p_{3} y_{1} / 122 h y y_{3} p_{2}$
X $65 p r y_{3} p_{2} / 121 p_{4}$ me $w$
XI $124 h y y_{2} p_{1} / 121 p_{4}$ me $w$
XII $65 p r y_{3} p_{2} / 46$ me $w$

* Diploids are symbolized by the symbol of the heterokaryon from which they are derived, but with the + replaced by $/$. The Roman numerals in front of the symbol give the code numbers of the diploids.

Table 4. Colour of sporing surface in parent strain, heterokaryons and heterozygous diploids

\begin{tabular}{|c|c|c|c|c|c|}
\hline \multicolumn{2}{|c|}{ Parent strains* } & \multicolumn{2}{|c|}{$\begin{array}{l}\text { Colour of conidia } \\
\text { in parent strains }\end{array}$} & \multirow{2}{*}{$\begin{array}{c}\text { Colour of } \\
\text { sporing surface } \\
\text { of } a+b \\
\text { heterokaryons }\end{array}$} & \multirow{2}{*}{$\begin{array}{l}\text { Colour } \\
\text { of conidia } \\
\text { of } a / b \\
\text { diploids }\end{array}$} \\
\hline$a$ & $b$ & $a$ & $b$ & & \\
\hline $63 y_{1}$ & $46 w$ & Yellow† & White & Yellowish white & Grey-green $\ddagger$ \\
\hline $63 y_{1}$ & $65 y_{3}$ & Yellow & Yellow & Yellow & Yellowish green \\
\hline $63 y_{1}$ & $122 y_{3}$ & Yellow & Yellow & Yellow & Yellowish green \\
\hline $63 y_{1}$ & $121 w$ & Yellow & White & Yellowish white & Grey-green \\
\hline $63 y_{1}$ & $124 y_{2}$ & Yellow & Yellow & Yellow & Yellow \\
\hline $124 y_{2}$ & $65 y_{3}$ & Yellow & Yellow & Yellow & Yellowish green \\
\hline $124 y_{2}$ & $121 w$ & Yellow & White & Yellowish white & Grey-green \\
\hline $65 y_{3}$ & $121 w$ & Yellow & White & Very pale yellow & Grey-green \\
\hline $65 y_{3}$ & $46 w$ & Yellow & White & Off-white & Grey-green \\
\hline
\end{tabular}

* Symbols not concerned with colour are omitted.

$\dagger$ All the yellow strains assume a tinge of pink after a time.

$\ddagger$ All the green strains tend to turn brown after a time.

conidia. Diploids between two yellow strains are yellowish-green in colour, except in the case of 63/124; five diploids established between a yellow and a white strain were of a grey-green colour slightly paler than the wild type (see Table 4). 
Penicillin production. Qualitative data relative to penicillin production on agar by heterokaryons and by heterozygous diploids agree in every case. With the strains used, heterokaryons and diploids between a producing strain and a non-producing strain produced penicillin; heterokaryons and diploids between two non-producing strains did not produce appreciable amounts of penicillin.

Table 5 gives quantitative results for penicillin production in submerged culture of heterozygous diploids as compared with the wild type (47.1564 Wis.) and with a diploid homozygous for penicillin production obtained from two strains derived from the wild type (26le/29 pr).

Table 5. Penicillin production in submerged culture by heterozygous diploids compared with that by strain 47.1564 Wis. (haploid)

Average yields of penicillin in three flasks shaken on rotary shaker are given. The standard errors are given as well as the values. Medium: corn steep + lactose. Precursor added every $12 \mathrm{hr}$. from the $24 \mathrm{hr}$.

\begin{tabular}{lcl} 
& & \multicolumn{1}{c}{$\begin{array}{c}\text { Penicillin } \\
\text { production } \\
\text { (units } / \mathrm{ml} .\end{array}$} \\
47. 1564 Wis. & at $\mathbf{9 6} \mathrm{hr}$ )
\end{tabular}

* Only symbols concerning the gene $p /+$ are given.

$\dagger$ Parent strains: 26 le (leucine) and $29 \mathrm{pr}$, both derived from 47.1564 Wis.

\$ Parent strains: 63 cy $p_{3} y_{1}$ and 51 pr thi (thiamine), which also derives from 47.1564 . Wis.

The results of submerged culture tests give substantial confirmation of the agar tests. Some $p / p$ diploids produce traces of penicillin, but the maximum is four units compared with the $\mathbf{4 1 4}$ units of the wild type control. The two $+/ p$ heterozygotes produce considerable quantities of penicillin. The diploid III $+1+$ produces about the same as the haploid wild type.

\section{Segregants from heterozygous diploids}

Segregants for colour or nutritional requirements have been isolated (Sermonti, 1954b) from all the strains described as heterozygous diploids in the present paper. This definitely confirms their heterozygosis.

Table 7 gives the results of a detailed segregation analysis carried out on the diploids VII 63 cy $p_{3} y_{1} / 46$ me w and XIII $63 c y p_{3} y_{1} / 121 p_{4}$ me $w$. The only difference between the two diploids is that XIII contains the allele $p_{4}$, which 
distinguishes strain 121 from its parent strain 46 . The segregants were selected for colour of conidia according to the method already described (Sermonti, $1954 b)$.

In both experiments it was found that the yellow segregants either were prototrophic or had a cysteine requirement, while the white segregants either were prototrophic or had a methionine requirement. All the yellow segregants from diploid VII were non-producing and all the white segregants produced penicillin. Neither white nor yellow segregants from diploid XIII produced penicillin.

Extensive experiments were carried out in an attempt to select a penicillinproducing segregant from a non-producing diploid. Diploid $\mathbf{X} 65$ pr $y_{3} p_{2} /$ $121 p_{4}$ me $w$ was tried first. Out of about 6000 colonies subjected to a preliminary test, and forty-two further checked, not a single penicillin-producing segregant was obtained.

The work was continued using the diploid XIII 63 cy $p_{3} y_{1} / 121 p_{4}$ me w. Out of 14,174 colonies tested a single penicillin-producing colony (308.6906) was detected. The penicillin production of this strain was tested in submerged culture (Table 6) and gives results similar to those of diploid VII.

Table 6. Submerged culture penicillin production by strain 308.6906 compared with production by diploid VII and strain 47.1564 Wis.

Average yields of penicillin in three flasks shaken on rotary shaker are given. The standard errors are given as well as the values. Medium: corn steep-lactose. Precursor added every $12 \mathrm{hr}$. from the $24 \mathrm{hr}$.

\begin{tabular}{llc}
\multicolumn{1}{c}{ Strain } & Yield of penicillin (units $/ \mathrm{ml}$. ) \\
\cline { 2 - 3 } 308.6906 & At $96 \mathrm{hr}$. & At $120 \mathrm{hr}$. \\
VII $46 / 63$ & $340 \pm 17$ & $369 \pm 2$ \\
47.1564 Wis. & $297 \pm 10$ & $249 \pm 11$ \\
& $489 \pm 11$ & $556 \pm 20$
\end{tabular}

The isolated strain proved to be green and prototrophic. It was genetically analysed by an examination of its second-order segregants selected for colour. Table 7 gives the details of the experiment.

It appears from Table 7 that, unlike the segregants of diploid VII, all the yellow segregants of this strain, and none of its white segregants, produced penicillin.

Some of the white segregants are prototrophic and some require methionine, but no yellow segregant was found with a cysteine requirement. The cysteine requirement was also looked for in segregants selected for nutritional requirements (Sermonti, 1954b). 426 out of the 1440 colonies examined were tested for nutritional requirements: 73 showed methionine requirement, but not one showed a cysteine requirement. Two yellow prototrophic segregants originating from strain 308.6906 were investigated for further segregation, but not one colony with a cysteine requirement appeared after testing 455 colonies selected from 3252. 
Table 7. Segregants from heterozygous diploids VII and XIII and from strain 308.6906 of Penicillium chrysogenum.

The segregants were selected for colour, purified and then tested for nutritional requirements and penicillin production.

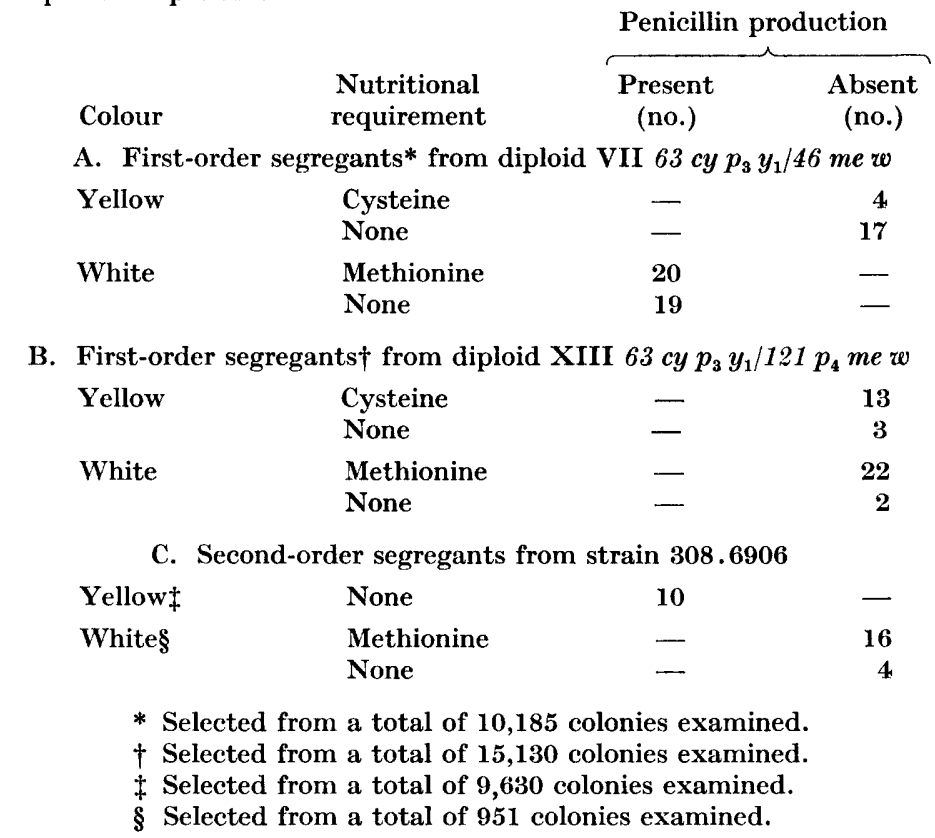

\section{DISCUSSION}

The genetic determination of the difference between presence and absence of capacity to produce penicillin is clear from the results given above, and in particular from the data on the segregation of diploid VII and of strain 308.6906. Absence of penicillin production $(p)$ segregates from penicillinproducing heterozygous diploids as determined by a single difference in a chromosome region. Absence is recessive to presence of penicillin production. Quantitative tests in submerged culture of the penicillin production of strains segregating from these diploids have not been carried out, but the evidence of the qualitative tests on agar cultures is sufficient indication of this segregation. Of the colour-selected segregants some showed no trace of production, while others showed rates of production similar to those of the parent type.

Linkage of the character $p$ with other characters may be deduced from the segregation data of diploid VII. In terms of mitotic segregation (Pontecorvo, Tarr Gloor \& Forbes, 1954) these data would mean that $p$ is distal to $y$, which would be in turn distal to $c y$.

The proportion of $p$ mutants- $0.25 \%$ in the present work, $0.20 \%$ in Bonner's (1947) work - is very high, considerably higher than the proportion of any mutant for a specific nutritional requirement obtained in Penicillium chrysogenum. At least three of the four $p$ alleles $\left(p_{1}\right.$ or $p_{2}, p_{3}$ and $\left.p_{4}\right)$ are 
certainly the result of independent mutation, since they were obtained in differently marked strains (see Table 1 ). $p_{1}, p_{3}$ and $p_{4}$ determine phenotypes which are slightly different (though $p_{1}$ and $p_{2}$ are phenotypically indistinguishable). Mutant $p_{3}$, which determines complete absence in the diploids $p_{3} / p$, also determines complete absence of penicillin in the haploid and reduced production in the $p_{3} /+$ diploids (see Table 5 ).

In diploids synthesized between pairs of non-producing strains the four $p$ behave as if they were allelic: i.e. practically no penicillin is produced by any of the six combinations between different non-penicillin-producing strains. Similarly, two mutations for yellow colour of conidia, $y_{1}$ and $y_{2}$, behave as if they were allelic. The green colour of the conidia does not reappear in diploid $\mathrm{XV}$, which is heterozygous, $y_{1} / y_{2}$. However, the third yellow mutant, $y_{3}$, is not allelic to the two just mentioned because the heterozygous diploids VI $y_{1} / y_{3}$ and XI $y_{2} / y_{3}$ have green conidia like the wild type. Whether all the $p$ mutants and two of the $y$ mutants, $y_{1}$ and $y_{2}$, should be called alleles or pseudo-alleles - considering, amongst other things, the possible origin of 308.6906 by crossing-over-is a matter of definition, and is not worth while discussing at the present stage of genetic analysis of Penicillium chrysogenum. On whatever interpretation, all the mutants studied which block penicillin synthesis concern a single gene. Two such genes, inactivated by different mutations, could not restore penicillin production when together in a heterozygote or in a heterokaryon.

The authors wish to express their thanks to Dr G. Pontecorvo, F.R.S., for his many valuable criticisms offered in the course of the work and in revising this paper; also to Mr F. G. Miles for the English translation of the Italian text.

\section{REFERENCES}

Bonner, D. (1947). Studies on the biosynthesis of penicillin. Arch. Biochem. $13,1$.

Camici, L., Sermonti, G. \& Chain, E. B. (1952). Observations on Penicillium chrysogenum in submerged culture. Bull. World Hlth Org. 6, 265.

Fries, N. (1948). Viability and resistance of spontaneous mutations in Ophiostoma representing different degrees of heterotrophy. Physiol. Plantar. 1, 330.

Jarvis, F. G. \& Johnson, M. J. (1950). The mineral nutrition of Penicillium chrysogenum Q 176. J. Bact. 59, 51.

Pontecorvo, G. \& Sermonti, G. (1953). Recombination without sexual reproduction in Penicillium chrysogenum. Nature, Lond. 172, 126.

Ponteconvo, G. \& Sermonti, G. (1954). Parasexual recombination in Penicillium chrysogenum. J. gen. Microbiol. 11, 94.

Pontecorvo, G., Tarr Gloor, E. \& Forbes, E. (1954). Analysis of mitotic recombination in Aspergillus nidulans. J. Genet. 52, 226.

Raper, A., Dorothy, F. A. \& Coghill, R. D. (1944). Natural variation and penicillin production in Penicillium notatum and allied species. J. Bact. 48, 639.

Roegner, F. R., Stahmann, M. A. \& Stauffer, J. G. (1954). Induction of variants in Penicillium chrysogenum by methyl-bis( $\beta$-chloroethyl)amine. Amer. J. Bot. 41, 1.

Roper, J. A. (1952). Production of heterozygous diploids in filamentous fungi. Experientia, 8, 14. 
Schmidt, W. H. \& Moyer, A. Y. (1944). Penicillin. I. Methods of assay. J. Bact. $47,199$.

Sermonti, G. (1954a). Genetics of Penicillium chrysogenum. I. Heterokaryosis in Penicillium chrysogenum. R.C. Ist. Sup. Sanit. (English edition), 17, 213.

Sermonti, G. (1954b). Genetics of Penicillium chrysogenum. II. Segregation and recombination from a heterozygous diploid. R.C. Ist. Sup. Sanit. (English edition), 17, 231.

Stahmann, M. A. \& Stauffer, J. F. (1947). Induction of mutants in Penicillium notatum by methyl-bis( $\beta$-chloroethyl)amine (nitrogen mustard). Science, 106, 35.

(Received 18 July 1955) 